\title{
Cardiovascular Fluctuations and Transfer Function Analysis in Stable Preterm Infants
}

\author{
PETER ANDRIESSEN, ANDRÉ M.P. KOOLEN, RALPH C.M. BERENDSEN, PIETER F.F. WIJN, \\ EDITH D.M. ten BROEKE, S. GUID OEI, AND CARLOS E. BLANCO \\ Neonatal Intensive Care Unit [P.A., A.M.P.K.], Department of Clinical Physics [R.C.M.B., P.F.F.W.], \\ Department of Obstetrics [E.D.M.t.B., S.G.O.], Máxima Medical Center, Veldhoven, The Netherlands; and Division \\ of Neonatology, Department of Pediatrics [C.E.B.], University of Maastricht, Maastricht, The Netherlands, and \\ Department of Physics, Eindhoven University of Technology, Eindhoven, The Netherlands [P.F.F.W.]
}

\begin{abstract}
To examine the baroreceptor reflex function, a beat-to-beat analysis between systolic blood pressure (SBP) and R-R interval fluctuations was studied in 10 stable appropriate-for-gestational age preterm infants (range, 27.2-33.7 wk) in the first postnatal week during quiet sleep. Spectral power analysis, using fast Fourier transform, and transfer functions (gain and phase difference) between SBP and R-R fluctuations were estimated in a low-frequency band (LF, 0.03-0.2 Hz) and high-frequency band (HF defined as the frequency band between the 10th and 90th centiles of the individual respiratory frequency). The LF/HF ratio reflects the sympathovagal balance. The mean frequency $( \pm \mathrm{SD})$ of LF peaks was centered at $0.07 \pm 0.02 \mathrm{~Hz}$. The mean frequency $( \pm \mathrm{SD})$ of the individual HF band was $0.82 \pm 0.21 \mathrm{~Hz}$. The LF/HF ratio in the R-R interval series [median, 29; interquartile range (IQR), 16-40] was higher than in the SBP series (median, 8; IQR, 4-14). The gain between R-R interval and SBP fluctuations (median, $4.2 \mathrm{~ms} / \mathrm{mm} \mathrm{Hg}$; IQR, 2.4-5.0) in the LF band was higher than in the HF band (median, $1.7 \mathrm{~ms} / \mathrm{mm} \mathrm{Hg}$; IQR, 1.4-3.0). SBP fluctuations lead R-R interval fluctuations in the $\mathrm{LF}$ band with a median phase difference of $+96^{\circ}$ (IQR,
\end{abstract}

\section{ABSTRACT}

67-132). At LF the fluctuations in SBP precede changes in R-R interval with a time delay of $3.8 \mathrm{~s}$. These observations indicate a dominant role of the sympathetic system in stable preterm infants in comparison with published adult values. Cross-spectral analysis allows a test for tracking the development of the sympathetic system in neonates. (Pediatr Res 53: 89-97, 2003)

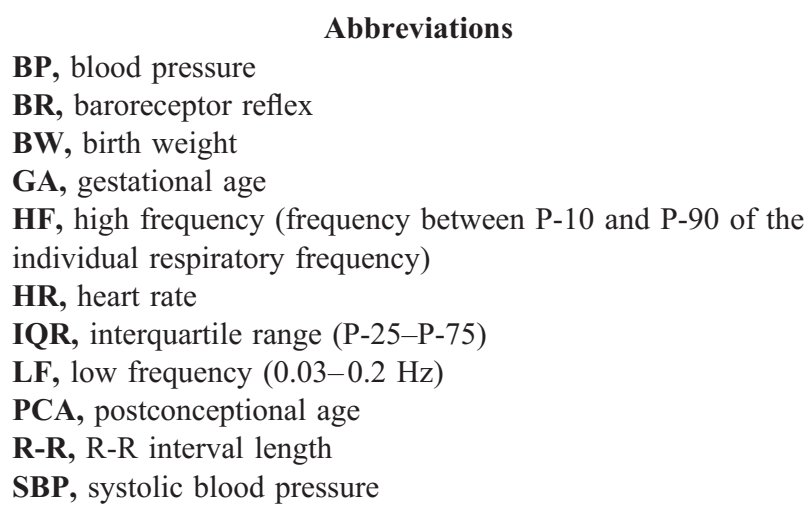

\section{Abbreviations}

The autonomic nervous system plays a key role in BP control by adapting HR and total peripheral resistance. Multiple physiologic mechanisms are responsible for fluctuations in $\mathrm{HR}$ and BP: respiration, BR, and thermoregulation. Power spectral analysis of the R-R interval series permits us to evaluate aspects of cardiovascular autonomic control (1). A HF spectral peak occurs at the respiratory frequency. A LF spectral peak, synchronous with Mayer waves in BP, occurs around 0.1 $\mathrm{Hz}$ and is attributed to the BR (2). Very low frequency oscillations (below $0.03 \mathrm{~Hz}$ ) are ascribed to peripheral resistance fluctuations caused by thermoregulation. HF fluctuations

Received November 21, 2001; accepted April 17, 2002.

Correspondence: Peter Andriessen, M.D., Máxima Medical Center, Neonatal Intensive Care Unit, Veldhoven, PO Box 7777, 5500 MB, The Netherlands; e-mail: pandriessen@sjz.nl

DOI: 10.1203/01.PDR.0000041514.73559.7D are associated with the parasympathetic system, whereas LF fluctuations are related to sympathetic and parasympathetic activity (3). The LF/HF ratio reflects the sympathovagal balance (3).

The BR can be considered a closed-loop circuit: BP fluctuations affect R-R interval fluctuations and vice versa (4). Spectral analysis technique and BR activation through postural change enable the assessment of BR gain/sensitivity in human adults $(5,6)$. Cardiovascular reflexes using spectral power analysis of R-R interval series have been studied in preterm and full-term infants (7-11). The LF/HF ratio progressively decreases with postnatal age, indicating an increase in parasympathetic contribution to control $\operatorname{HR}(8,9,11)$. In general, these findings suggest that in preterm infants BR becomes more functional with postnatal development. To our knowledge cross-spectral analysis of BP and R-R interval fluctuations have not been examined earlier in preterm and term 
infants for the estimation of BR sensitivity. This stimulated our interest in evaluating the cardiovascular autonomic control in stable preterm infants by studying the relationship between spontaneous fluctuations of invasively measured BP and R-R interval signals. The objectives of this investigation were to determine the feasibility of cross-spectral analysis of R-R interval and SBP series, evaluating the sympathovagal balance ( $\mathrm{LF} / \mathrm{HF}$ ratio) in both R-R intervals series and SBP series, and assessing the transfer function (BR gain/sensitivity and phase) in stable preterm infants.

\section{METHODS}

Subjects. The study group consisted of 10 stable preterm infants. The GA ranged from 27.2 to $33.7 \mathrm{wk}$, and $\mathrm{BW}$ ranged from 800 to $2050 \mathrm{~g}$. Six infants were born spontaneously, and four were delivered by cesarean section. All infants received prenatal steroids. The 5-min Apgar ranged between 5 and 10, and the umbilical arterial blood $\mathrm{pH}$ ranged between 7.2 and 7.4. All infants were appropriate-for-gestational age, according to the Dutch growth charts (12). All infants were studied once in the immediate postintensive care phase between 2 and $8 \mathrm{~d}$ of postnatal life. Arterial catheters were neither inserted nor remained longer in place because of the study. The PCA ranged from 28.1 to $34.3 \mathrm{wk}$. All infants were breathing room air spontaneously. There were no signs or symptoms of asphyxia, respiratory distress, sepsis, or patency of the ductus arteriosus at the time of the study. None had echoencephalographic evidence of cerebral hemorrhage or parenchymal lesions. Echocardiography revealed no structural abnormalities. All subjects received caffeine therapy (blood levels, $11-17 \mathrm{mg} / \mathrm{L}$ ) to prevent apnea. Caffeine was administered once a day at midnight. Recordings were made at least $12 \mathrm{~h}$ after caffeine administration. Cardiotropic drugs were not used. Values of electrolytes, blood gas analysis, and hematocrits were all within normal range. Informed consent was obtained from the parents of each infant. The study was approved by the local ethics committee.

Data acquisition. A bipolar chest lead of the surface ECG and the transthoracic electric impedance waveforms were recorded by Hewlett Packard neonatal monitor type Merlin (Waltham, MA, U.S.A.). Arterial BP was measured invasively through a $4 \mathrm{~F}$ catheter placed earlier in the lower aortic position for routine monitoring of vital functions and intensive care management. A $0.5-\mathrm{mL} / \mathrm{h}$ infusion of heparinized physiologic saline solution was continuously flushed through the catheter.

Recordings were made in the prone position for $1-2 \mathrm{~h}$. Data segments were selected subsequently during periods of regular breathing and spontaneous sleep with closed eyes and without gross body movements (quiet sleep state) (13). Data analysis was performed on 192-s-long stationary segments. Because preterm infants have an immature and irregular respiratory drive, 3-min segments were chosen as a compromise between the demands of sufficient duration and signal stability. The quality of the signals was visually verified for artifacts, ectopic beats, baseline drifts, apnea periods, or gross bradycardia.

Frequency ranges of interest. The whole frequency band of interest was the range between 0.03 and $2 \mathrm{~Hz}$. The very low frequency band $(0-0.03 \mathrm{~Hz})$ was discarded to avoid possible contribution of slow trend artifacts. In addition, fast Fourier transform has a limited accuracy of power estimation in this frequency range using records of relatively short duration. The LF band was defined as the frequency range between 0.03 and $0.2 \mathrm{~Hz}$. Because the HF band primarily contains the reflection of respiratory activity, the HF band was individualized for each subject depending on his or her respiratory drive. The respiration rate was estimated by peak detection of the thoracic waveforms resulting in a mean value and a bandwidth between the 10th and 90th centiles (P-10 and P-90, respectively) of the breath-by-breath frequency. The frequency range between these centile values was used to identify each subject's individual HF band. The upper spectral limit of the HF band (the 90th centile of the respiratory frequency) was less than half of the mean HR, thereby meeting the requirements of the Nyquist critical frequency. An example of a spectral power density curve is shown in Figure 1.

Data analysis. The sample frequency was $400 \mathrm{~Hz}$ for the ECG signals and $100 \mathrm{~Hz}$ for the arterial BP and transthoracic electric impedance signals. The sampling error of $\mathrm{R}$ top detection was optimized using a second-order polynomial fit, resulting in an accuracy of $\mathrm{R}$ top detection of $0.2 \mathrm{~ms}$. $\mathrm{R}$ waves were detected from the ECG, and an unevenly spaced R-R interval sequence was created. This sequence was resampled using a BoxCar window at $4 \mathrm{~Hz}$ to obtain an equidistantly spaced time series (14). The mean R-R interval was calculated and subtracted from all data points to remove the direct current component. SBP was identified from peak detection of the BP signal, resulting in an unevenly spaced "systogram." The systogram was converted into an equidistantly spaced time series using the same resampling method as used for the R-R interval time series.

Each 192-s-long segment of preprocessed evenly spaced R-R interval and SBP series was subdivided into five halfoverlapping 64-s (256 points) segments. To reduce the effects of spectral leakage each segment was multiplied with a triangular Parzen window (15). Fast Fourier transform was used to compute the auto- and cross-spectral density functions for each segment. The spectral resolution was $0.016 \mathrm{~Hz}$. Filter correction removed unwanted side effects of the earlier resampling and Parzen windowing. A mean discrete amplitude spectrum was derived from the five autospectral density functions. Powers in the ranges of interest were calculated from the obtained mean autospectral density functions. The total power was computed for the frequency band between 0.03 and $2 \mathrm{~Hz}$. The values for power are presented in units of square milliseconds (for R-R interval series) and square millimeters of mercury (for SBP series). The LF and HF were expressed as normalized units [e.g. LF/total power $\times 100 \%]$ (3).

Accuracy of power estimation. Using 64-s segments (resulting in a spectral resolution of $0.016 \mathrm{~Hz}$ ) and five halfoverlapping segments, power estimation above $0.03 \mathrm{~Hz}$ is accurate. R-R interval and SBP series can be considered as a combination of noise-like and signal-like data with a Gaussian distribution. The SD of the value per frequency bin is $100 \%$ of the calculated power spectral density $(16,17)$. Averaging five 

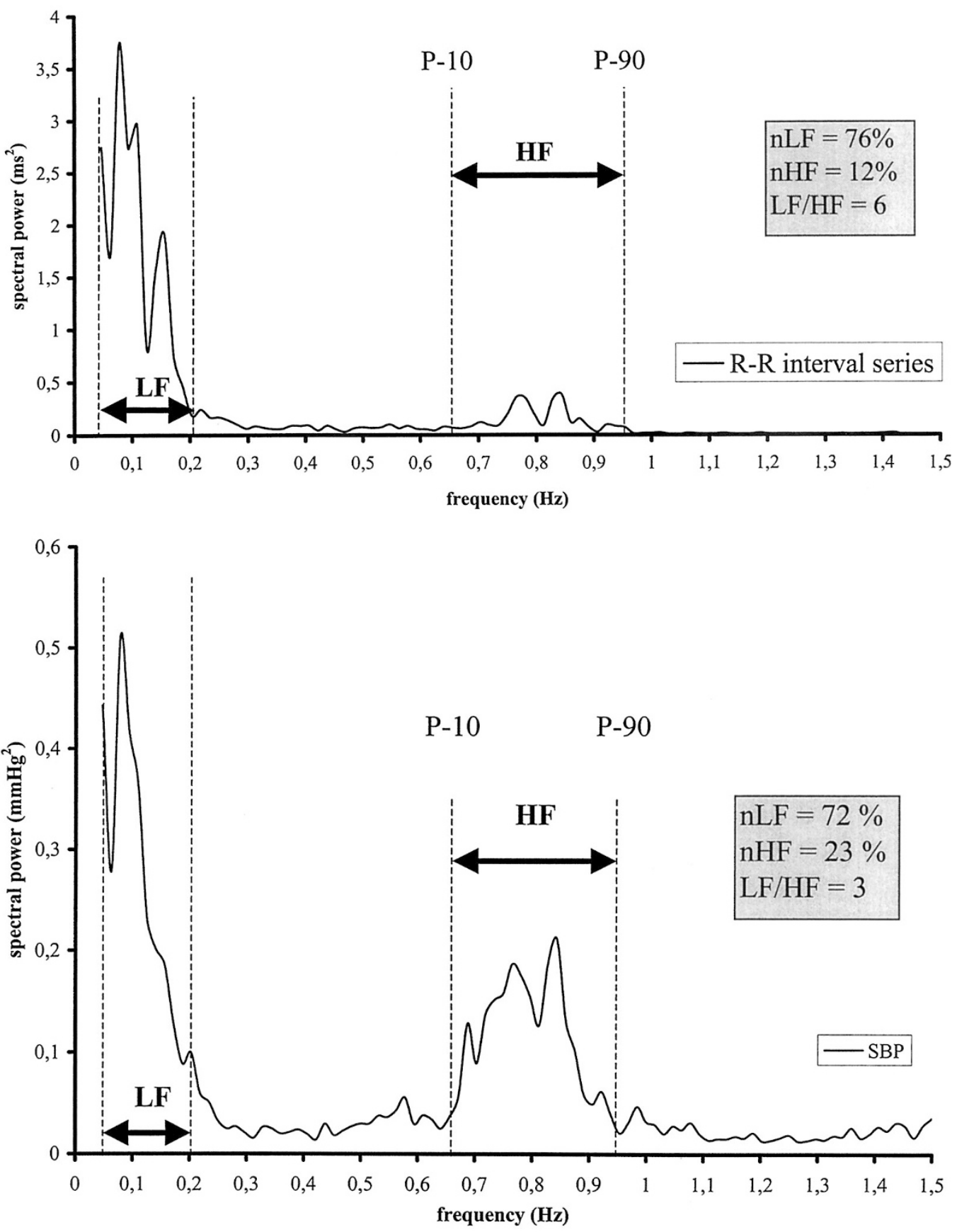

Figure 1. Spectral power curves of R-R interval series and systolic blood pressure series of a spontaneously breathing infant at a PCA of 30 wk. Top, spectral power curve of R-R interval series. The LF band was defined between 0.03 and $0.2 \mathrm{~Hz}$ and is marked between the vertical lines. A clear LF peak was centered at $0.08 \mathrm{~Hz}$. The individual HF band was defined between the P-10 and P-90 value of the respiratory frequency and is marked between the vertical lines. A modest HF peak was determined around $0.8 \mathrm{~Hz}$ and ranged between 0.65 (P-10) and 0.95 (P-90) Hz. LF and HF are expressed as normalized values (nLF $=$ LF/total power $\times 100 \%$ and $\mathrm{nHF}=\mathrm{HF} /$ total power $\times 100 \%$ ) and $\mathrm{LF} / \mathrm{HF}$ value (unitless). Bottom, spectral power curve of SBP. The LF peak for SBP was centered at $0.08 \mathrm{~Hz}$. Note the much more pronounced peak in the HF band, resulting in a lower LF/HF value.

spectra leads to a relative SD of the mean amplitude magnitude per frequency bin of $23 \%$.

Transfer function analysis. The amount of linear coupling between two signals in the frequency domain can be expressed by means of the (squared) coherence function (18). This measure is comparable to the regression analysis in the time domain, except that it is computed for each frequency region. The coherence function, which ranges between 0 (no relationship) and 1 (linear relationship), was used to assess the statis- tical reliability of the transfer function estimates at each frequency. Only data in which the coherence was above 0.5 were used in the estimation of the transfer functions. The transfer functions (gain and phase) between SBP and R-R interval signals were estimated from the average auto- and crossspectral density functions. Gain and phase were assessed in the LF and HF bands at the frequency of highest coherence. The gain (milliseconds per millimeter of mercury) reflects the degree to which the input signal (BP) amplitude becomes 
manifest in the output signal (R-R interval) amplitude at a discrete frequency. The phase difference (degrees) indicates the lead or lag of one signal with respect to the other at a discrete frequency (Figs. 2 and 3).

Spontaneous BR gain/sensitivity. The transfer function gain was used to estimate frequency-specific BR sensitivity during normal physiologic fluctuations (19-21). In addition a time domain estimate was used to describe BR sensitivity. The SD of $(\mathrm{R}-\mathrm{R})_{\mathrm{i}}$ intervals (square root of variance or root-meansquare for the mean) and SBP beat-to-beat values were determined in the 192-s-long segments. As variance is related to the total power of spectral analysis, the ratio of SD of $(\mathrm{R}-\mathrm{R})_{\mathrm{i}}$ intervals divided by the SD of SBP (milliseconds per millimeter of mercury) was used as a time domain estimate for total variability gain (22).

Statistical analysis. Data with a normal distribution are expressed as a mean $\pm \mathrm{SD}$, otherwise data are expressed as a median and IQR. Comparisons were made with (two-sided) paired $t$ test for parametric data and the Wilcoxon sign rank test for nonparametric data. The influence of GA, PCA, and BW on spectral power values and transfer function variables was studied by linear regression analysis, using the method of least squares, and ANOVA. Statistical significance was accepted with $p$ values less than 0.05 .

\section{RESULTS}

The clinical characteristics of the study population are shown in Table 1. From the 192-s-long segments of each infant the mean and SD of the R-R interval, SBP, and diastolic BP were calculated.
A representative example of R-R interval spectral power and SBP spectral power of a spontaneously regular-breathing preterm infant (mean respiratory frequency, $0.8 \mathrm{~Hz} ; \mathrm{P}-10,0.65 \mathrm{~Hz}$; and P-90, $0.95 \mathrm{~Hz})$ at a PCA of $30 \mathrm{wk}$ is shown in Figure 1. In the spectra the following features are characteristic: 1 ) a higher peak in the LF band compared with the HF band, both in R-R interval and SBP power spectrum; 2) the LF peak is centered around 0.08 $\mathrm{Hz}$, both in R-R interval and SBP power spectrum; and 3) a clear HF peak in the SBP power spectrum and a less pronounced HF peak in the R-R interval power spectrum.

Table 2 displays the resulting spectral power values and the $\mathrm{LF} / \mathrm{HF}$ ratios for the study group. In all subjects LF and HF peaks could be determined. The mean frequency of the LF peak was $0.07 \pm 0.02 \mathrm{~Hz}$. In all subjects an individual respiratory frequency rate (mean and P-10, P-90 centile boundaries of the individual HF band) could be calculated. The mean frequency of the individual HF band was $0.82 \pm 0.21 \mathrm{~Hz}$. Normalized LF power in R-R interval series (median, 74\%; IQR, 70-76) were significantly higher than SBP series (median, 60\%; IQR, 50-64). In contrast, the normalized HF power in R-R interval series (median, 3\%; IQR, 2-5) was significantly lower than SBP series (median, 7\%; IQR, 4-14). The LF/HF ratio in R-R interval series (median, 29; IQR, 16-40) was significantly higher than in SBP series (median, 8; IQR, 4-14).

Figures 2 and 3 illustrate examples of the cross-spectral transfer function analysis between R-R interval and SBP signals of the same preterm infant as in Figure 1.

The mean and SD of coherence between R-R interval and SBP in the LF band was $0.75 \pm 0.13$, whereas coherence between R-R interval and SBP in the HF band was $0.83 \pm$

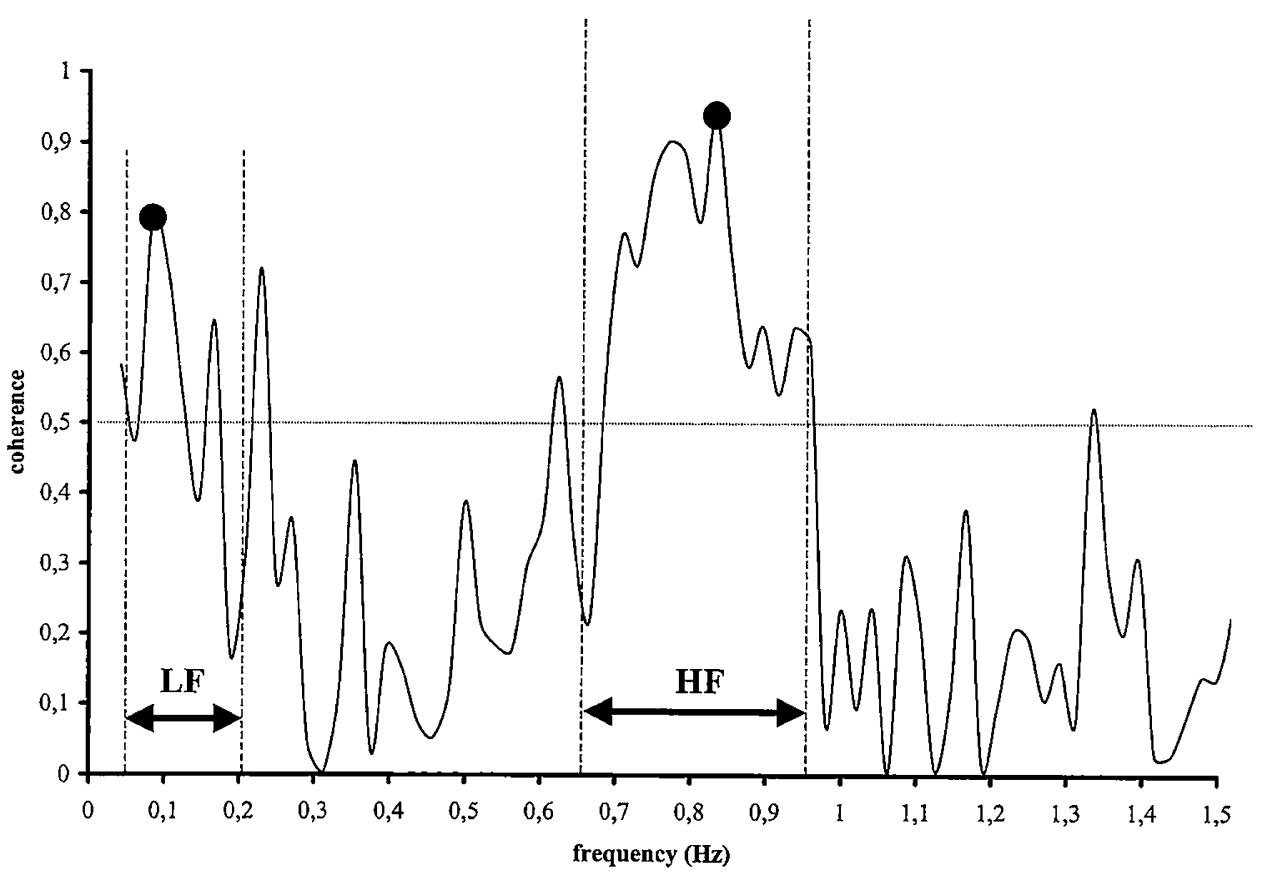

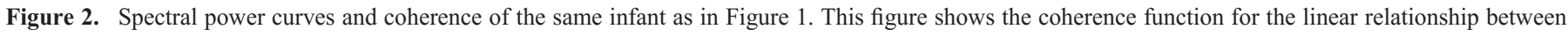

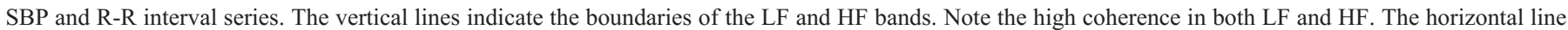

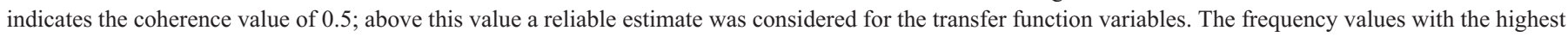
coherence in LF $(0.08 \mathrm{~Hz})$ and HF $(0.83 \mathrm{~Hz})$ were chosen (indicated as dots) to compute gain and phase (Fig. 3). 

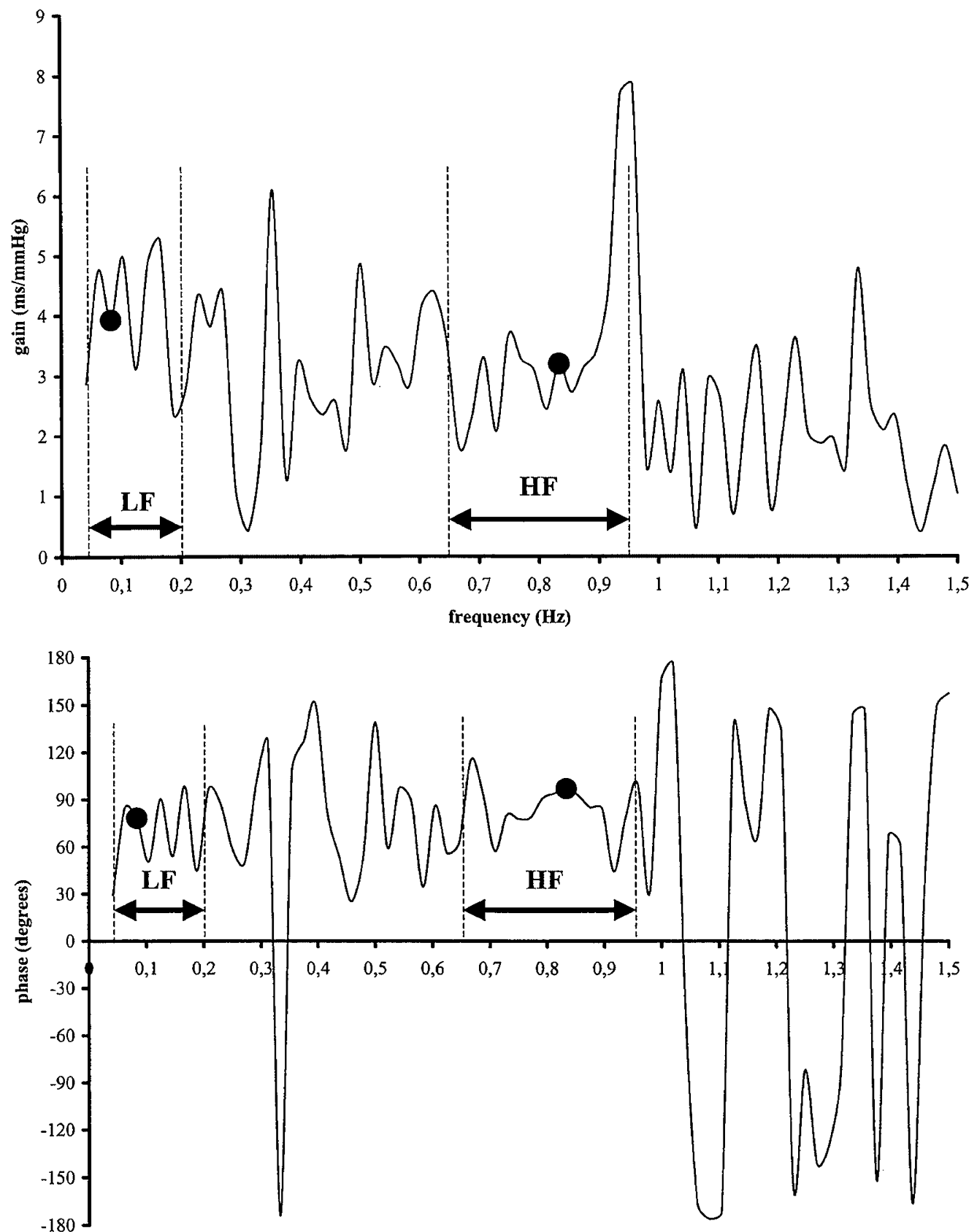

frequency $(\mathbf{H z})$

Figure 3. Transfer function parameters of the same infant as in Figure 1. Top, gain as function of frequency. The vertical lines indicate the boundaries of the LF and HF bands. The indicated dots (LF, $3.9 \mathrm{~ms} / \mathrm{mm} \mathrm{Hg} ; \mathrm{HF}, 3.2 \mathrm{~ms} / \mathrm{mm} \mathrm{Hg}$ ) correspond with the frequency values with the highest coherence (see Fig. 2). Bottom, phase as function of frequency. The phase spectrum has positive values if BP fluctuations leading HR fluctuations. The vertical lines indicate the boundaries of the LF and HF bands. The indicated dots (LF, $78^{\circ}$; HF, $97^{\circ}$ ) correspond with the frequency values with the highest coherence (see Fig. 2).

0.09. The high coherence between R-R interval and SBP implicates reliable estimates of the transfer functions gain and phase. The gain between R-R interval and SBP (median, 4.2 $\mathrm{ms} / \mathrm{mm} \mathrm{Hg}$; IQR, 2.4-5.0) in the LF band was higher in the HF band (median, $1.7 \mathrm{~ms} / \mathrm{mm} \mathrm{Hg}$; IQR, $1.4-3.0 ; p<0.05$ ). At LF the phase difference was positive for all subjects; a median phase difference of $+96^{\circ}$ (IQR, 67-132) was calculated. At HF the phase difference was positive (BP fluctuations leading HR changes; $n=7$ ) or negative (BP fluctuations following HR changes; $n=3$ ), a median phase difference of $+87^{\circ}(\mathrm{IQR},-85$ to +94 ) was calculated. Mean R-R interval and mean SBP were not related to any transfer function value.

The median value of the ratio of $S D$ of $(R-R)_{i}$ intervals divided by the SD of SBP beat-to-beat values was $3.2 \mathrm{~ms} / \mathrm{mm}$ $\mathrm{Hg}$ (IQR, 2.3-4.5). Figure 4 shows the correlation between LF cross-spectral and time domain gain. LF cross-spectral analysis correlated significantly with the time domain assessment of spontaneous BR sensitivity $\left(r^{2}=0.70 ; y=1.1 x-0.4 ; p<\right.$ $0.01)$. HF cross-spectral analysis did not show a significant correlation with the time domain index. 
Table 1. Clinical characteristics and time domain measures of the study population

\begin{tabular}{lc}
\hline Gestational age (wk) & $30.2 \pm 2.1$ \\
Birthweight (g) & $1369 \pm 395$ \\
Postnatal age (d) & $5.1 \pm 1.4$ \\
R-R interval (ms) & $432 \pm 36$ \\
SBP (mm Hg) & $59 \pm 8$ \\
DBP (mm Hg) & $35 \pm 5$ \\
\hline
\end{tabular}

Data are expressed as mean $\pm \mathrm{SD}$.

Systolic blood pressure (SBP); diastolic blood pressure (DBP).

Table 2. Spectral power values of the study population

\begin{tabular}{lcccc}
\hline & $\begin{array}{c}\text { R-R } \\
\text { interval } \\
\text { series }\end{array}$ & SBP series & $\begin{array}{c}p \\
\text { value* }\end{array}$ \\
\hline Total power & $31(28-59)$ & $3.7(2.7-4.8)$ & \\
LF power & $21(18-44)$ & $1.9(1.6-3.5)$ & \\
HF power & $2(1-3)$ & $0.3(0.2-0.6)$ & \\
Normalized LF power (\%) & $74(70-76)$ & $60(50-64)$ & $<0.005$ \\
Normalized HF power (\%) & $3(2-5)$ & 7 & $(4-14)$ & $<0.005$ \\
LF/HF ratio & $29(16-40)$ & 8 & $(4-14)$ & $<0.05$ \\
\hline
\end{tabular}

Spectral powers in the ranges of interest were calculated from the obtained mean autospectral density functions for R-R interval series and systolic blood pressure (SBP) series. The values for spectral power (Total, LF, and HF power) are presented in units of $\mathrm{ms}^{2}$ (for R-R interval series) and $\mathrm{mm} \mathrm{Hg}^{2}$ (for SBP series). In addition the LF and HF were expressed as normalized values (for example: $\mathrm{LF} /$ total power $\times 100 \%$ ). LF/HF values are unitless.

Data are expressed as median and interquartile range. * Wilcoxon sign rank test for non-parametric data.

The total power was computed for the frequency band between 0.03 and 2 $\mathrm{Hz}$. The low frequency (LF) band was defined between $0.03-0.2 \mathrm{~Hz}$. The high frequency $(\mathrm{HF})$ band was defined as the frequency range between the P-10 and P-90 centile of the individual respiratory frequency.

No clear influence of GA, PCA, BW, and type of delivery (spontaneous or cesarean section) could be demonstrated on spectral power values, LF/HF ratios, and the transfer function variables.

\section{DISCUSSION}

Our data demonstrate for the first time that cross-spectral analysis of R-R interval series and beat-to-beat SBP series is

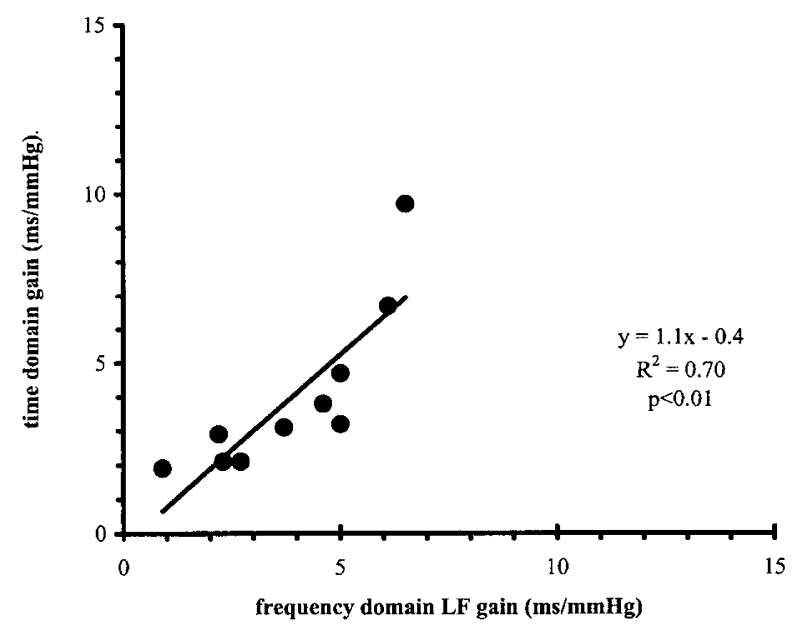

Figure 4. Correlation diagram between cross-spectral LF gain and time domain gain. Correlation between cross-spectral LF gain and time domain estimate for total variability gain [SD of $(\mathrm{R}-\mathrm{R})_{\mathrm{i}}$ interval series divided by the $\mathrm{SD}$ of systolic beat-to-beat $\mathrm{BP}$ values]. feasible in stable preterm infants. Some of these aspects are different from human adult data (3-6). First, our results show high LF/HF ratios in both R-R interval series and beat-to-beat SBP series during resting conditions. Second, the LF peaks of $\mathrm{R}-\mathrm{R}$ interval and SBP fluctuations are centered around $0.07 \mathrm{~Hz}$. Third, the magnitude of the transfer function (BR gain/ sensitivity) between R-R interval and SBP is higher in the LF range (approximately $4 \mathrm{~ms} / \mathrm{mm} \mathrm{Hg}$ ) than in the $\mathrm{HF}$ range (approximately $2 \mathrm{~ms} / \mathrm{mm} \mathrm{Hg}$ ). In accordance with human adult data $(4,18)$, we found a consistent positive phase difference between SBP and R-R interval fluctuations in the LF range. However, our data suggest that preterm infants have a longer time delay of the baroreceptor loop. We found that crossspectral LF (BR) gain correlates significantly with a time domain estimate for total variability gain.

These observations do not allow us to discuss the participation of the autonomic nervous system in preterm infants in response to abnormal situations (hypotension or hypertension). It would be interesting to know whether more mature preterm infants present a better adaptation capability during pathologic conditions. These studies could be difficult to perform as they will be based on challenging the BR loop with different maneuvers to increase or decrease BP. This of course could be pursued using animal models with all their limitations as it is very difficult to work with very premature animal models.

$\boldsymbol{L F} / \boldsymbol{H F}$ ratio. The $\mathrm{LF} / \mathrm{HF}$ ratio in human adults is close to 1 during resting supine condition (3). We found, both in R-R interval and SBP series, much higher LF/HF ratios. In addition, the higher LF/HF ratio in R-R interval series compared with SBP series was striking. In R-R interval series, normalized values of LF power were higher than in SBP series, whereas normalized values of HF power were lower in R-R interval series than in SBP series. Despite differences in methodology (for example, definition of frequency bands) our LF/HF ratio values are in the same range as that found by others $(8,9,11)$. The high LF/HF ratio in preterm infants during quiet sleep could be interpreted as a predominance of sympathetic influence or immature parasympathetic activity. GA has been shown to correlate with decreasing LF/HF ratio in R-R interval series with advancing GA $(9,11)$. The decreasing ratio could be consistent with an increase of parasympathetic influence. A low parasympathetic influence in preterm infants could lead to a situation in which the relatively fast respiratory-induced BP changes cannot be buffered by modulations of the R-R interval. Only the parasympathetic system is fast enough to influence cardiovascular variables in the frequency range of respiration in preterm infants. The consequence is a relatively high level of $\mathrm{BP}$ lability in the HF range, resulting in a lower LF/HF ratio in SBP series than in R-R interval series. In fetal lambs breathing movements were associated with strong fluctuations in BP and weaker fluctuations in HR (23). In addition it is speculated that early in life changes in HR do not contribute to a great degree in reflex control of circulation (24). However, the differences of HF variability of the two signals are much more complex, because in fetuses the lungs are full of liquid and thus fetal breathing movements are not comparable to the breathing movements of neonates. Still, these fetal findings are in accordance with our observations. 
The LF peak. In all subjects LF peaks could be determined, and the mean of LF fluctuations was centered around $0.07 \mathrm{~Hz}$ (cycle length, 14.3 s). This value is in good agreement with an earlier study in neonates concerning HR variability (25). In adults the spectral peak in the LF range is around $0.1 \mathrm{~Hz}$, corresponding to the so-called 10 -s rhythm $(3,26)$. We hypothesize that the natural frequency of the baroreceptor control loop in preterm infants is lower than in adults. However, we have to keep in mind that entrainment of the baroreceptor loop is not easy in these tiny infants. We are developing research to explore this hypothesis further by challenging the BR through tilt testing by measuring simultaneously HR, beat-to-beat SBP, and blood flow. Relatively low conductivity in the immature nervous system could be responsible for a longer time delay in the BR loop, resulting in a lower natural frequency of the control loop.

The magnitude of the transfer function (BR gain/ sensitivity). The gain was calculated for frequencies with a coherence greater than 0.5 , indicating a linear dependency between R-R interval and SBP signals. In our study population of preterm infants the cardiovascular variability was particularly present in the LF range. This resulted in a higher gain of the transfer function between R-R interval and SBP series in the LF range, compared with that in the HF range. In the LF range the gain was approximately $4 \mathrm{~ms} / \mathrm{mm} \mathrm{Hg}$, and in the $\mathrm{HF}$ range approximately $2 \mathrm{~ms} / \mathrm{mm} \mathrm{Hg}$. Gain calculated for human

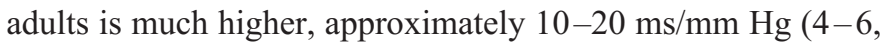
27-29). However, in contrast to our findings, the HF gain in adults is higher than the LF gain $(4,5,29)$. Assuming a higher vagal activity in adult life during rest one might expect higher gain in the HF range compared with the LF range in adults. Higher LF gain, however, might indicate a sympathetic predominance in preterm infants. From spontaneous, nonrespiratory-related SBP and R-R interval variations, a BR sensitivity of $4 \pm 2 \mathrm{~ms} / \mathrm{mm} \mathrm{Hg}$ was assessed in preterm infants (30). In fetal lambs a gain of $4 \mathrm{~ms} / \mathrm{mm} \mathrm{Hg}$ was assessed using norepinephrine to increase BP (31). Data in neonatal rabbits and lambs showed the relationships between BP and R-R interval fluctuations are greatest at lower frequencies $(19,32,33)$. Despite differences in methodology and species, the results of these (neonatal) studies are in accordance with our data and differ from human adult data. To our knowledge no comparative transfer function data are available in full-term infants. The reason for this lack of data is unclear, but may be related to the fact that small preterm infants have arterial lines for monitoring and blood withdrawal, whereas term babies with lines are mostly asphyxiated or in extreme respiratory distress and do not represent normal physiology.

The phase of the transfer function. The phase of the transfer function indicates the temporal relationship between the signals in the frequency domain $(6,19)$. However, in case of an intact baroreceptor closed-loop system, judgment considering causality and interpretation of the phase spectrum is hampered, because it is not clear whether one signal leads another by $360^{\circ}+n$ degrees or is behind by $360^{\circ}-n$ degrees. The phase difference of $+96^{\circ}$ at $0.07 \mathrm{~Hz}$ (cycle length, $14.3 \mathrm{~s}$ ) represents a time delay of $3.8 \mathrm{~s}$. We interpreted this as at LF, changes in BP lead the change in R-R interval for the following reasons. First, consistent BR activity is R-R interval lengthening in response to an increase in BP, or R-R interval shortening in response to a decrease in BP (30). Second, the temporal relationship between the signals, illustrated in Figure 5, strongly suggests LF fluctuations in SBP precede fluctuations in R-R intervals for about the calculated time delay of approximately $4 \mathrm{~s}$. It is highly unlikely that at LF, R-R interval changes precede arterial pressure changes with a latency of $14.3-3.8=10.5 \mathrm{~s}$.

In human adults, a LF peak at $0.10 \mathrm{~Hz}$ and a phase difference of approximately $60-70^{\circ}$ (pressure leads R-R interval) are calculated $(4,6,34)$. This corresponds with a far lesser time delay between BP and R-R interval fluctuations in human adults, approximately $2 \mathrm{~s}$. These findings suggest that preterm infants have a longer time delay of the baroreceptor loop.

In case of HR frequencies of $2.5 \mathrm{~Hz}$ and respiratory frequencies of approximately $0.8 \mathrm{~Hz}$, taking into account the closed loop properties, interpretation of the phase shift in the HF range is not possible. We did not found a consistent positive or negative phase difference at HF. It is not clear whether HF fluctuations in SBP lead or lag fluctuations in R-R intervals (Fig. 5).

Frequency domain (transfer function) versus time domain assessment of BR gain. Transfer function can be used effectively to estimate the BR sensitivity provided that the phase relationship and coherence between variables are considered $(4,19-21)$. We found a strong and significant correlation between frequency-specific transfer function gain in the LF band and a time domain estimate for total variability gain. To our knowledge this has never been demonstrated before. Timedomain analysis of variability refers to statistical calculations

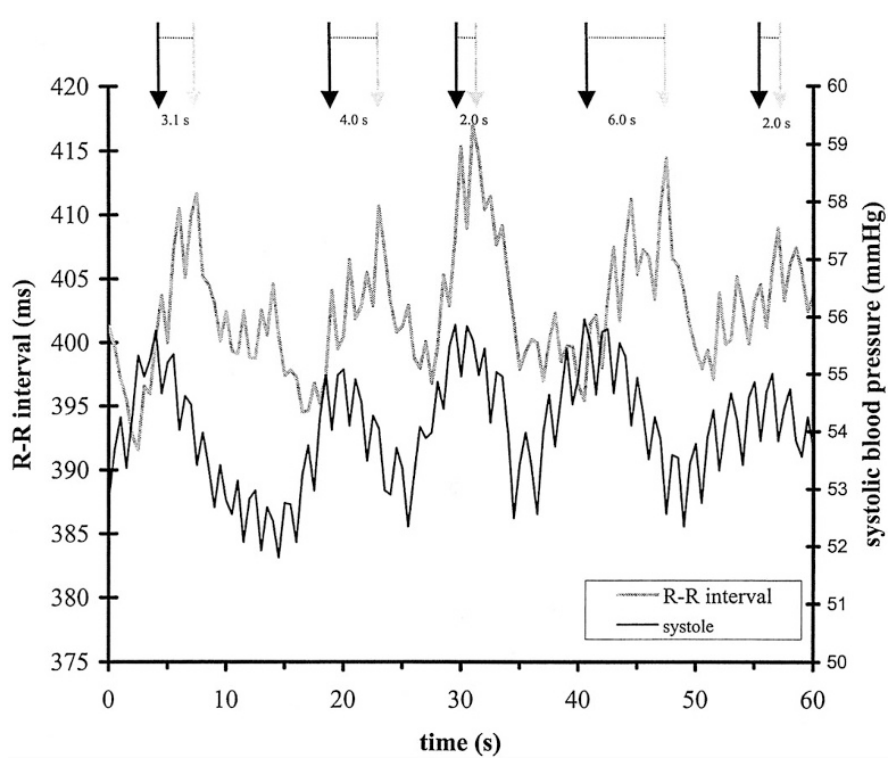

Figure 5. Temporal relationship between SBP and R-R interval of the same infant as in Figure 1. R-R interval (left $y$ axis) and SBP (right $y$ axis) values are shown as a function of time ( $x$ axis). Time is expressed in seconds. Besides HF fluctuations, related to the respiratory frequency, approximately five LF fluctuations in this trace of $60 \mathrm{~s}$ can be seen from the signals, corresponding with an LF peak of approximately $0.08 \mathrm{~Hz}$. LF fluctuations of BP precede BR changes in R-R intervals with a time delay varying between 2 and $6 \mathrm{~s}$ (period between arrows). 
of adjacent (e.g. R-R interval values) intervals, e.g. modifications of the mean, SD, sorting and selecting, and slope changes (35). An example of a simple time domain index is the SD of $(\mathrm{R}-\mathrm{R})_{\mathrm{i}}$ intervals within a period. This index has been thought to result from the dynamic balance between the sympathetic and parasympathetic components of the autonomic nervous system (36). The SD of (R-R $)_{i}$ intervals or SBP-to-SBP values within a certain period reflects the total variance of the signal. Power spectral density analysis provides the basic information of how power (variance) distributes as a function of frequency. Because LF power has been demonstrated to be dominant in both $\mathrm{R}-\mathrm{R}$ and SBP frequency analysis, it is not surprising that the BR gain derived from cross-spectral analysis correlates strongly with a time domain estimate for total variability gain. In other words BR gain/sensitivity in the LF range can be estimated by a simple time domain index.

Methodological considerations. The small size of the group and the range in GA in our study population explains the lack of evidence of the effect of GA, PCA, and BW on any of the spectral power values or the transfer function variables in the study group. Another possible limitation of the study might be the effect of antenatal administration of steroids on glucocorticoid-dependent maturation of the BR and fetal HR variability. Antenatal glucocorticoids decrease the sensitivity of the BR after birth in a preterm delivered sheep model (37). In human fetuses antenatal glucocorticoids transitorily lower short- and long-term fetal HR variability for 1-3 d after administration and normalize at $\mathrm{d} 4$ (38). Although the period between antenatal glucocorticoid administration and postnatal measurement was at least $5 \mathrm{~d}$ in this study, it is possible that our postnatal results might reflect the effect of antenatal steroids rather than normal development of the preterm infant. Another issue concerns the physiologic significance of HR variability with respect to autonomic nervous system and maturation. Inasmuch as HR variability reflects the response of the sinoatrial node to the effects of the autonomic nervous system, it is possible that specific receptors of the sinoatrial node are not mature at the same time in preterm infants. HR variability may thus not necessary reflect the direct input of the autonomic system.

\section{CONCLUSION}

In conclusion, we studied cardiovascular variability in stable preterm infants by means of cross-spectral analysis of R-R interval and SBP fluctuations. We found high $\mathrm{LF} / \mathrm{HF}$ ratios in both R-R interval series and SBP series in preterm infants. The magnitude of the transfer function (BR gain or sensitivity) between R-R interval and SBP is higher in the LF band than in the HF band. This suggests that a high LF/HF ratio, reflecting an altered sympathovagal balance, might be a result of increased sympathetic activity. Our findings suggest a longer time delay of LF fluctuations between BP and R-R interval in preterm infants (approximately $4 \mathrm{~s}$ ) than in human adults (approximately $2 \mathrm{~s}$ ). The cross spectral signal analysis is an interesting technique because it could offer a test for tracking the development of the sympathetic system.
Acknowledgment. The authors thank Ben Janssen (Department of Pharmacology, Cardiovascular Research Institute of Maastricht, University of Maastricht, Maastricht, The Netherlands) for his critical review of the manuscript.

\section{REFERENCES}

1. Akselrod S, Gordon D, Madwed JB, Snidman NC, Shannon DC, Cohen RJ 1985 Hemodynamic regulation: investigation by spectral analysis. Am J Physiol 249:H867-H875

2. Penáz J 1978 Mayer waves: history and methodology. Automedica 2:135-141

3. Task Force of the European Society of Cardiology and the North American Society of Pacing and Electrophysiology 1996 Heart rate variability: standards of measurement, physiological interpretation, and clinical use. Circulation 93:1043-1065

4. de Boer RW, Karemaker JM, Strackee J 1987 Hemodynamic fluctuations and baroreflex sensitivity in humans: a beat-to-beat model. Am J Physiol 253:H680-H689

5. Saul JPh, Berger RD, Albrecht P, Stein SP, Hui Chen M, Cohen RJ 1991 Transfer function analysis of the circulation: unique insights into cardiovascular regulation. Am J Physiol 261:H1231-H1245

6. Cooke WH, Hoag JB, Crossman AA, Kuusela TA, Tahvanainen KUO, Eckberg DL 1999 Human responses to upright tilt: a window on central autonomic integration. J Physiol (Lond)517:2: 617-628

7. ten Voorde BJ, Ree EJ, Hack WWM, Bergschneider VM, Hoekstra BPT, Faes ThJC, Witte H, Rother M 1992 High-frequency and low-frequency heart-rate fluctuations analysis in newborns: a review of possibilities and limitations. Basic Res Cardiol 87:193-204

8. van Ravenswaay-Arts C, Hopman J, Kollée L, Stoelinga G, van Geijn H 1994 Spectral analysis of heart rate variability in spontaneously breathing very preterm infants. Acta Paediatr 83:473-480

9. Chatow U, Davidson S, Reichman BL, Akselrod S 1995 Development and maturation of the autonomic nervous system in premature and full-term infants using spectral analysis of heart rate fluctuations. Pediatr Res 37:294-302

10. Scher MS, Steppe DA, Dokianakis G, Sun M, Guthrie RD, Sclabassi RJ 1994 Cardiorespiratory behavior during sleep in full-term and preterm neonates at comparable postconceptional term ages. Pediatr Res 36:738-744

11. Mazurksky JE, Birkett CL, Bedell KA, Ben-Haim SA, Segar JL 1998 Development of baroreflex influences on heart rate variability in preterm infants. Early Hum Dev $53: 37-52$

12. Kloosterman GJ 1970 On intrauterine growth. Int J Gynaecol Obstet 8:895-912

13. Prechtl HFR 1974 The behavioural states of the newborn infant (a review). Brain Res 76:185-212

14. Jaffe RS, Fung DL 1994 Constructing a heart rate variability analysis system. J Clin Monit 10:45-58

15. Akselrod S 1995 Components of heart rate variability: basic studies. In: Malik M, Camm AJ (eds) Heart Rate Variability. Futura Publishing, New York, pp 147-163

16. Welch PD 1967 The use of fast Fourier transform for the estimation of power spectra: a method based on time averaging over short, modified periodograms. IEEE Trans Audio Electroacoust 15:70-73

17. Bingham C, Godfrey MD, Tukey JW 1967 Modern techniques of power spectrum estimation. IEEE Trans Audio Electroacoust 15:56-66

18. de Boer RW, Karemaker JM, Strackee J 1985 Relationships between short-term blood pressure fluctuations and heart rate variability in resting subjects: a spectral approach. Med Biol Eng Comput 23:352-358

19. Head GA, Lukoshkova EV, Burke SL, Malpas SC, Lambert EA, Janssen BJA 2001 Comparing spectral and invasive estimates of baroreflex gain. IEEE Eng Med Biol Mag 20:43-52

20. Robbe HWJ, Mulder LJM, Rüddel H, Langewitz WA, Veldman JBP, Mulder G 1987 Assessment of baroreceptor reflex sensitivity by means of spectral analysis. Hypertension 10:538-543

21. Honzíková N, Fišer B, Honzík J 1992 Noninvasive determination of baroreflex sensitivity in man by means of spectral analysis. Physiol Res 41:31-37

22. Smeulders M, Agbeko R, de Jong W, Koolen AMP 1994 Modulation of heart rate by the arterial baroreceptor reflex in neonates. Pediatr Res 212:38A(abstr)

23. Metsälä T, Siimes A, Antila K, Välimäki I 1993 Association of breathing movements to the variability of heart rate and blood pressure in foetal lambs. Acta Physiol Scand 147:213-219

24. Blanco CE, Dawes GS, Hanson MA, McCooke HB 1988 Carotid baroreceptors in fetal and newborn sheep. Pediatr Res 24:342-346

25. Giddens DP, Kitney RI 1985 Neonatal heart rate variability and its relation to respiration. J Theor Biol 113:759-780

26. Saul JPh, Rea RF, Eckberg DL, Berger RD, Cohen RJ 1990 Heart rate and muscle sympathetic nerve variability during reflex changes of autonomic activity. Am J Physiol 258:H713-H721

27. Fritsch JM, Eckberg DL, Graves LD, Wallin BG 1986 Arterial pressure ramps provoke linear increases of heart period in humans. Am J Physiol 251:R1086-R1090

28. Sanders JS, Ferguson DW, Mark AL 1988 Arterial baroreflex control of sympathetic nerve activity during elevation of blood pressure in normal man: dominance of aortic baroreflexes. Circulation 77:279-288

29. Clayton RH, Bowman AJ, Ford GA, Murray A 1995 Measurement of baroreflex gain from heart rate and blood pressure spectra: a comparison of spectral estimation techniques. Physiol Meas 16:131-139

30. Drouin E, Gourmay V, Calamel J, Mouzard A, Rozé JC 1997 Assessment of spontaneous baroreflex sensitivity in neonates. Arch Dis Child 76:F108-F112 
31. Wakatsuki A, Murata Y, Ninomiya Y, Masaoka N, Tyner JG, Kutty KK 1992 Physiologic baroreceptor activity in the fetal lamb. Am J Obstet Gynecol 167:820827

32. Grönland JU, Antila KJ, Siimes ASI, Metsälä T, Oja R, Tuominen J, Välimäki IAT 1989 Beta-adrenergic control and inter-relationships between heart rate and blood pressure in neonatal lambs. Med Biol Eng Comput 27:163-170

33. Grönland JU, Kalli ST, Siimes ASI, Sydänmaa M, Antila KJ, Välimäki IAT 1991 Do $\beta$-adrenergic blockade and sleep state affect cardiorespiratory control in neonatal lambs? Multivariate autoregressive modeling approach. Pediatr Res 29:272-277

34. Baselli G, Cerutti S, Civardi S, Liberati D, Lombardi F, Malliani A, Pagani M 1986
Spectral and cross-spectral analysis of heart rate and arterial blood pressure variability signals. Comput Biomed Res 19:520-534

35. Parer WJ, Parer JT, Holbrook RH, Block BSB 1985 Validity of mathematical methods of quantitating fetal heart rate variability. Am J Obstet Gynecol 153:402-409

36. Cabal LA, Siassi B, Zanini B, Hodgman JE, Hon EE 1980 Factors affecting heart rate variability in preterm infants. Pediatrics 65:50-56

37. Segar JL, Lumbers ER, Nuyt AM, Smith OJ, Robillard JE 1998 Effect of antenatal glucocorticoids on sympathetic nerve activity at birth in preterm sheep. Am J Physiol 274:R160-R170

38. Derks JB, Mulder EJH, Visser GHA 1995 The effects of maternal betamethasone administration on the fetus. Br J Obstet Gynaecol 102:40-46 